\title{
ACQUA PER NUTRIRE IL PIANETA: LA SITUAZIONE MONDIALE
}

\author{
MARIA CRISTINA RULLI (*)
}

SuNTO. - L'aumento della popolazione mondiale e la conseguente domanda di cibo, acqua ed energia sta esercitando una crescente pressione su suolo, risorse idriche e ecosistemi. Le proiezioni delle Nazioni Unite indicano una popolazione di 8.3 miliardi nel 2030 e l'Agenzia Internazionale per l'Energia prevede un conseguente aumento del consumo di energia pari $30 \%$. Il consumo alimentare aumenterà del $50 \%$ con la popolazione a causa del cambiamento delle abitudini alimentari dei paesi emergenti e a forte crescita demografica. Tutta la produzione alimentare dipende direttamente o indirettamente dall'agricoltura alla quale attiene la maggior parte dell'attuale utilizzo di risorsa idrica dolce (circa l' $85 \%$ a scala mondiale). Solo una piccola frazione di acqua viene utilizzata per uso domestico ( $\approx$ $5 \%$ ), mentre circa il 10\% viene impiegata per usi industriali facenti direttamente uso di energia. La produzione energetica sia essa una fonte di tipo idroelettrico, nucleare, da combustibili fossili, da biomassa o da fratturazione dipende dalla disponibilità di risorsa idrica. Vi è una certa preoccupazione che nei prossimi decenni le risorse di acqua dolce disponibili sulla Terra potrebbero non essere sufficienti a soddisfare la crescente domanda di cibo, fibre e biocarburanti. La domanda che ci si pone è: è l'uomo in procinto di affrontare una grave crisi idrica? Alla fine del XVIII secolo Malthus (1798) osservò che il tasso di crescita demografica era maggiore di quello della produzione alimentare, prevedendo quindi che, nel lungo periodo, l'umanità non avrebbe più avuto risorse sufficienti per sfamare se stessa. Nelle sue analisi Malthus non considerava il notevole aumento di capacità di accesso al cibo, oltre che di produzione dello stesso, che sarebbe stato prodotto dai progressi tecnologici. La storia moderna e contemporanea è stata infatti contraddistinta dall'avvento di almeno tre importanti "rivoluzioni" che hanno aumentato la produzione alimentare: (1) la rivoluzione industriale agli inizi del 1800, (2) la così detta "rivoluzione verde" negli anni Cinquanta, e (3) l'intensificazione del commercio mondiale. Quest'ultima, particolarmente evidente negli ultimi decenni, ha consentito ad alcune società di poter fare affidamento su prodotti alimentari importati da altri paesi, in tal modo utilizzando virtualmente le risorse idriche disponibili nei paesi esportatori. Il commercio di prodotti alimentari e altre merci è infatti associato ad un trasporto di acqua virtuale dal luogo di produzione a quello di consumo. Il commercio dell'acqua virtuale è un meccani-

(*) Politecnico di Milano-Dipartimento di Ingegneria Civile e Ambientale, Milano, Italia. E-mail: mariacristina.rulli@polimi.it 
smo tramite il quale si può attuare una ridistribuzione delle risorse idriche e quindi di cibo, rendendo possibile che paesi ove la risorsa idrica ed alimentare è scarsa possano avere ciò di cui abbisognano importandolo da altri. Ciò malgrado, la crescita demografica in atto potrebbe spingere l'umanità verso i limiti imposti dalla limitatezza delle risorse. Anche se nell'immediato non vi è prova di una recente risposta demografica alle limitate risorse, tali limiti esistono e la disponibilità di risorse naturali controlla, in ultima analisi, la dimensione della popolazione mondiale. La crisi finanziaria e poi alimentare del 2008 ha evidenziato la prossimità dei limiti di risorse naturali nella quale ci troviamo ad operare. Essa ha infatti prodotto un consistente aumento dei prezzi alimentari, una politica protezionista dei paesi esportatori, una crisi nei paesi importatori e un conseguente aumento della popolazione affetta da malnutrizione (oltre un miliardo). La crisi del 2008 e gli episodi di instabilità politica che seguirono nel 2010-11 suggeriscono che la crescente domanda di acqua e cibo hanno eroso la capacità di resilienza della società verso le calamità naturali diminuendo la sicurezza alimentare e che una soluzione urgente per assicurare la sicurezza alimentare nei paesi dipendenti dall'importazioni è necessaria. Mentre soluzioni quali l'aumento di terra coltivata a spese degli "ecosistemi naturali" sono risultate poco sostenibili per il conseguente aumento delle emissioni di $\mathrm{CO} 2$ e perdita di biodiversità, colmare il divario tra la resa colturale potenziale ed effettiva nei terreni già coltivati, ma attualmente poco produttivi, sembra essere la soluzione più sostenibile. Negli anni immediatamente successivi alla crisi finanziara del 2008 alcune nazioni dipendenti dall'importazione di prodotti agricoli hanno iniziato ad investire in grandi estensioni di terreni agricoli situati per lo più nei paesi in via di sviluppo con notevole disponibilità di risorsa idrica e manodopera a basso prezzo, dove l'introduzione di nuove tecnologie poteva aumentare l'attuale resa agricola rendendo l'investimento redditizio. Tale soluzione pone degli importanti interrogativi sullo stato delle risorse idriche presenti e future: quanta risorsa idrica stiamo attualmente utilizzando? E' essa rinnovabile su degli orizzonti temporali fruibili? Di quanta risorsa idrica avremo bisogno per nutrire il pianeta nel futuro prossimo? Nel prossimo futuro quanta e dove sarà ubicata la risorsa idrica disponibile alla produzione di cibo? Quali sono i volumi idrici in gioco nel commercio di acqua virtuale? Quanto i paesi (sviluppati e in via di sviluppo) si affidano a tale commercio per soddisfare le proprie necessità? E' possibile pianificare lo sviluppo di un paese confidando sul commercio di acqua virtuale?

$* * *$

ABSTRACT. - Growing population and the associated increasing global demand for farmland products, energy and fibers is placing unprecedented pressure on soil, water resources and ecosystems. The UN population prospects shows 8.3 billion people in 2030 and the IEA outlook reports the $30 \%$ increase of the energy consumption. Compared with the present, agricultural products demand is expected to increase up to $50 \%$. Most of the human appropriation of freshwater resources is due to agricultural production (about $85 \%$ globally), while only a smaller fraction of water is used for household ( $\approx 5 \%$ ) or industrial needs $(\approx 10 \%)$. Because all food production depends directly or indirectly on agriculture, overall feeding the humanity requires much more water than quenching its thirst. There is some concern that in the next few decades the finite freshwater resources available on Earth might not be sufficient to meet the increasing human demand for food, fibers, and biofuels. Is mankind about to face a severe water crisis? At the end of the XVIII century Malthus (1798) noted that the rate of demographic growth tended to be faster than the increase in food supply, and argued that in the long run the humanity will not have enough resources to feed itself. Malthus' analysis, however, did not account for 
our ability to dramatically increase our access to food through major technological advances. The modern and contemporary history has seen at least three major changes that have increased food production: (1) the industrial revolution in the early 1800s; (2) the green revolution (with river damming, fertilizers and new cultivars) in the fifties; and (3) the intensification of global trade, which allows some societies to rely on food imported from other countries and to virtually use water resources available abroad. The trade of food and other commodities is associated with a virtual transport of water from production to consumption regions. Virtual water trade is an effective mechanism to alleviate regional food and water scarcity by virtually redistributing water resources thereby allowing a number of countries to have access to the food they need. However, demographic growth could eventually push the humanity against the "planetary boundaries" imposed by resource limitations. Even though there is no evidence of any recent demographic response to resource limitations, such boundaries ought to exist and natural resources should ultimately control the size of the global population. Signs of our proximity to these planetary boundaries were adverted during the 2008 food crisis, when food prices skyrocketed, some exporters banned food exports, trade-dependent countries panicked, and the number of undernourished people in the world exceeded 1 billion. The 2008 crisis and the episodes that followed in 2010-11 suggest that the increasing demand for water and food have eroded the societal resilience and increased food insecurity . A solution is urgently needed, particularly for import-dependent countries. While in most cases the long term benefits of putting more land under the plow at the expenses of "natural ecosystems" are not justified by the associated increase in $\mathrm{CO}_{2}$ emissions and biodiversity losses, closing the yield gap ("distance" between potential and actual agricultural yields) in the existing cultivated land appears to be a more viable approach to food security. This requires investments in agriculture that many countries - particularly those with relatively large yield gaps - cannot afford. Therefore, until recently some of the potential breadbaskets of the world (e.g., Ukraine, Sudan, Tanzania) have remained underutilized. In the last few years, prompted by the recent food crises and the escalating demand for freshwater resources worldwide, some of the import-dependent countries have started to invest in foreign agricultural land in regions where water availability, yield gaps, and the cost of land make the development of commercial farming a profitable activity. This solution brings important questions on the state of present and future water resources. How much water are we using? What about the need of water in the next future? The virtual water trade can be considered a solution for feeding the humanity in the next future?

Il nostro pianeta è attualmente in grado di utilizzare solo una piccola parte dell'acqua che dispone. Sulla terra è disponibile circa 1.7 Miliardi di $\mathrm{km}^{3}$ di acqua, il $97.5 \%$ della quale è salata e il restante $2.5 \%$ è dolce. Della frazione di acqua dolce presente sulla terra solo una parte di essa è direttamente utilizzabile poiché circa il $70 \%$ è allo stato solido (ghiacciai, coperture nivali permanenti). Acqua dolce allo stato liquido è presente nei corpi idrici superficiali e in quelli sotterranei rispettivamente per lo $0.3 \%$ e il $30.8 \%$ del totale di acqua dolce. La distribuzione spazio-temporale delle risorse idriche sul pianeta è disomogenea, risultando in aree di abbondanza ed aree di scarsità idrica. La scarsità 
idrica è definita come il rapporto fra il prelievo e la disponibilità di acqua e può essere declinata sia come scarsità idrica di tipo fisico, qualora la scarsità sia dovuta a fattori fisici cioè alla non disponibilità di acqua, sia come scarsità di tipo economico. Quest'ultimo tipo di scarsità idrica si manifesta qualora la risorsa idrica in un dato luogo esiste, ma non vi sono le infrastrutture per l'utilizzo della stessa (Fig. 1).

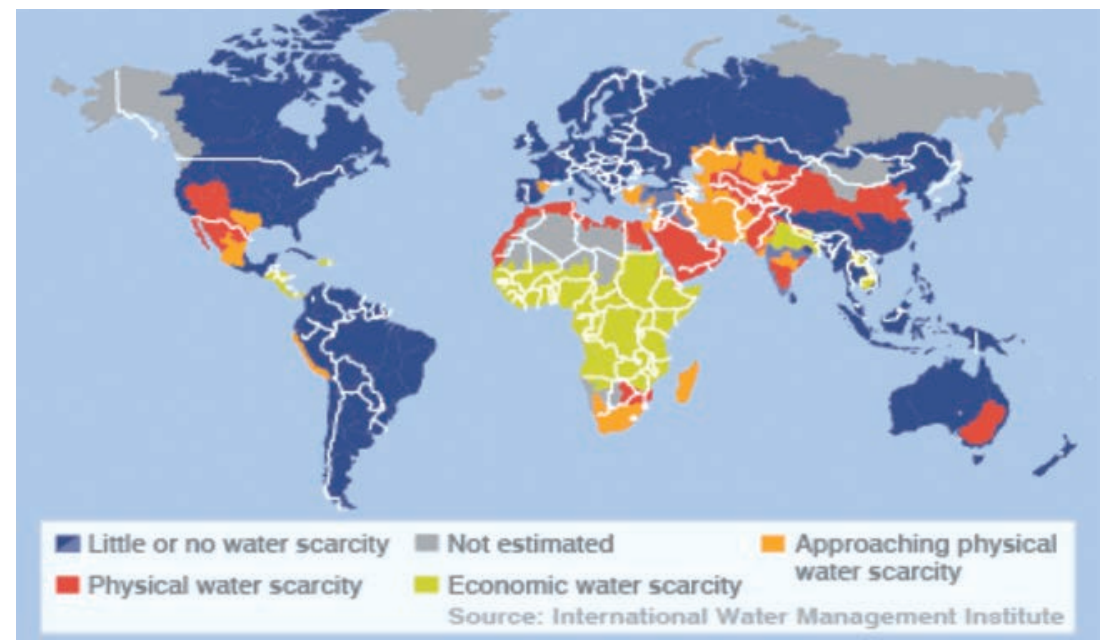

Fig. 1. Scarsità idrica di tipo fisico e scarsità idrica di tipo economico. (Fonte: International Water Managment Institute).

Gran parte dell'uso della risorsa idrica dolce è dovuto all'agricoltura, quasi $86 \%$, mentre il 9,6\% dell'acqua viene utilizzata per uso industriale, il 4,6\% per uso domestico. L'utilizzo dell'acqua per i diversi comparti non è spazialmente omogeneo; nei paesi in via di sviluppo gran parte dell'acqua viene utilizzata nel comparto agricolo, mentre nei paesi sviluppati, il comparto industriale e il comparto domestico coprono gran parte dell'uso dell'acqua.

Il principale uso delle risorse idriche d'acqua dolce quindi è quello alimentare. L'uso di acqua dolce procapite, inteso come l'acqua necessaria a produrre gli alimenti dei quali ci nutriamo, varia in relazione sia alla quantità del cibo che viene ingerito, sia alla tipologia degli alimenti costituenti la dieta. Un'analisi globale alla scala nazionale mostra come nel nostro pianeta l'acqua necessaria a produrre gli alimenti di una dieta giornaliera nazionale media vari dai 600 ai 2500 metri cubi per persona l'anno (Mekonnen E Hoekstra (2011)) (Fig. 2). Da ciò 
discende che alcuni paesi nel mondo mostrano avere carenza alimentare e malnutrizione ed altri una sovra alimentazione (Figg. 3 e 4).

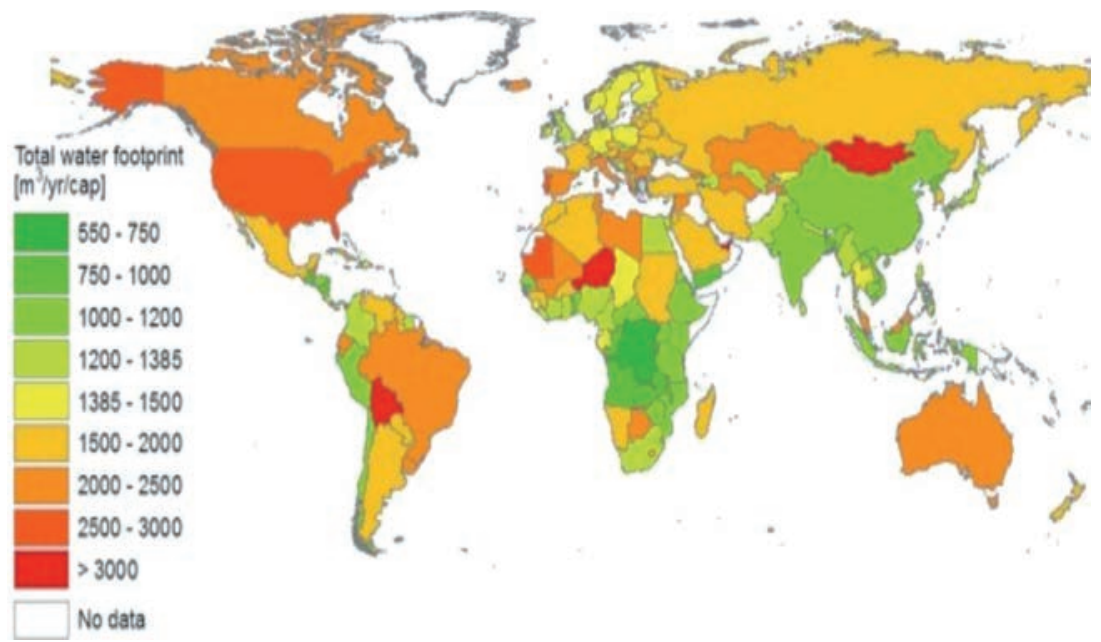

Fig. 2. Utlizzo d'acqua procapite annuo 1996-2005.

(Fonte: Mekonnen E Hoekstra, 2011).

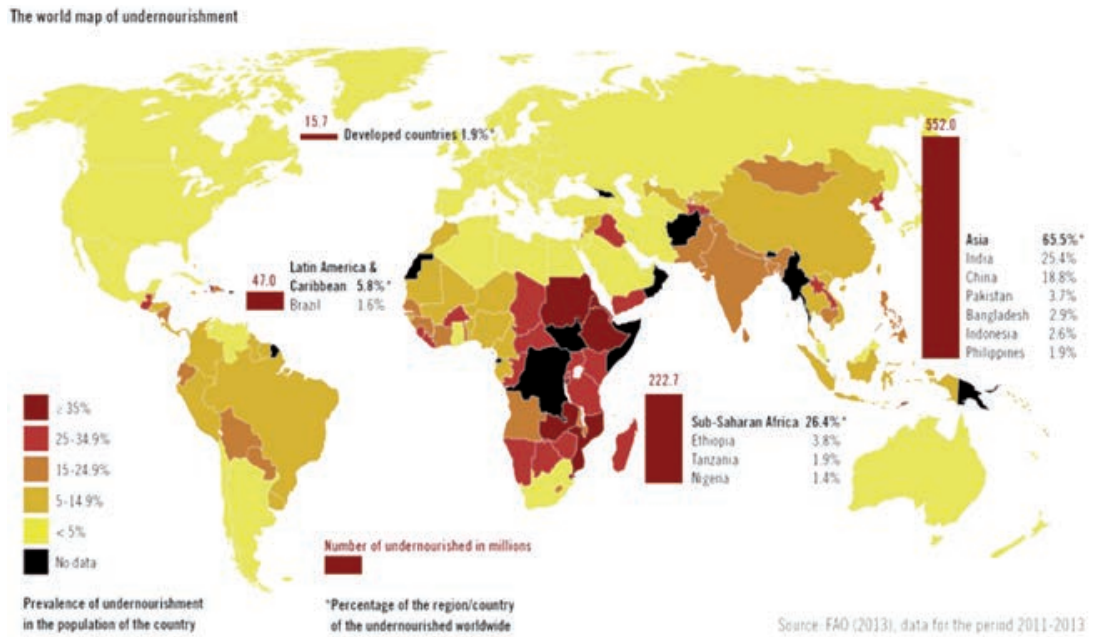

Fig. 3. Mappa mondiale della malnutrizione: prevalenza di sottonutrizione.

(Fonte: FAO, 2013) 


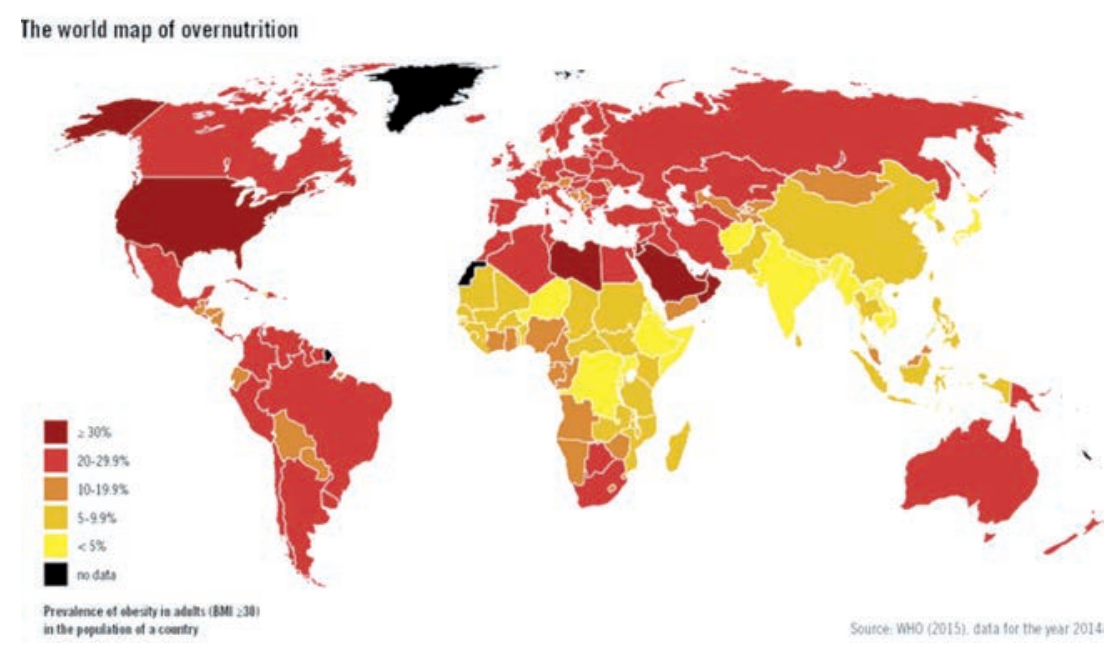

Fig. 4. Mappa mondiale della sovranutrizione: prevalenza dell'obesità negli adulti (BMI>30). (Fonte: WHO, 2015).

Le diverse diete adottate nei diversi paesi nel mondo necessitano di una quantità di acqua per la produzione degli alimenti che compongono tali diete variabile con la tipologia dei cibi stessi. Cibi di origine animale sono idricamente più esigenti di quelli vegetali. Di conseguenza, diete di tipo vegetariano o pescetariano necessitano di una quantità minore di quelle a base di carne.

Attualmente l'acqua utilizzata a scala mondiale per l'alimentazione è $1.2 \times 10^{13} \mathrm{~m}^{3}$ (D'Odorico et al., 2010) con una previsione di aumento del $60 \%$ della domanda di cibo al 2050 (Alexandratos and Bruinsma, 2012). Si prevede un aumento di utilizzo di risorsa idrica dolce ascrivibile ad una crescita della popolazione, ad un utilizzo di biomasse a scopo energetico e ad un cambiamento di abitudini alimentari di alcune popolazione ad alto tasso di crescita di GDP. Le proiezioni delle Nazioni Unite indicano una popolazione di 9.3 miliardi nel 2050 (UN DESA, 2011) e l'Agenzia Internazionale per l'Energia prevede un conseguente aumento del consumo di energia pari $30 \%$.

Poiché tutta la produzione alimentare dipende direttamente o indirettamente dall'agricoltura alla quale attiene la maggior parte dell'attuale utilizzo di risorsa idrica dolce e la produzione energetica sia essa una fonte di tipo idroelettrico, nucleare, da combustibili fossili, da biomassa o da fratturazione dipende dalla disponibilità di risorsa idrica 
vi è una certa preoccupazione che nei prossimi decenni le risorse di acqua dolce disponibili sulla Terra potrebbero non essere sufficienti a soddisfare la crescente domanda di cibo, fibre e biocarburanti.

Le domande che ci si pone sono:

- di quanto cibo e quindi risorsa idrica avremo bisogno nel prossimo futuro?

- Le risorse naturali disponibili sono sufficienti a soddisfare le necessità di cibo future?

- E' l'uomo in procinto di affrontare una grave crisi idrica?

Uno studio condotto da Folkemark e Rockstrom (2006) ha quantificato in $8700 \mathrm{~km}$ cubi il volume di risorsa idrica dolce necessario a soddisfare le esigenze alimentare di una popolazione di 9.5 miliardi e di un eradicamento della malnutrizione (presente nel 2006 in 800 milioni di individui nel mondo). Tali stime necessitano di un adeguamento alla luce delle proiezioni sui cambiamenti della dieta (Fig. 5a) e sull'incremento di utilizzo di energie da biomassa (Fig. 5b) che potrebbero rendere il volume stimato insufficiente alla produzione del cibo necessario alla popolazione mondiale al 2050. Sarà infatti necessaria una quantità di acqua maggiore rispetto a quella stimata da Folkemark e Rockstrom (2006) a causa sia dell' adozione da parte di alcune popolazioni di diete basate su cibi idricamente più esigenti rispetto ai cibi attualmente consumati, sia di un utilizzo di acqua per la produzione di biomasse utilizzate a scopo energetico (Figg. 5a e 5b).

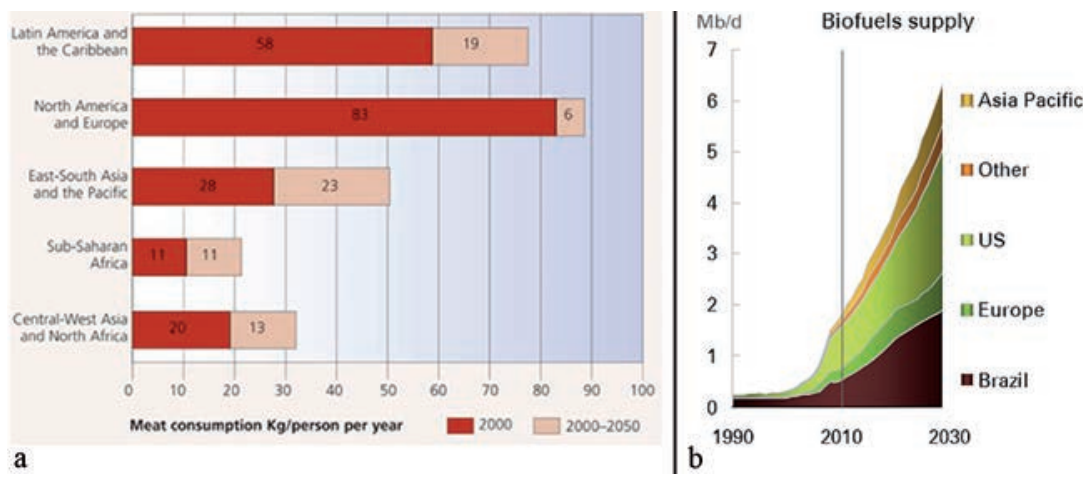

Fig. 5. a. Proiezione sui cambiamenti di dieta (Fonte FAO-OECD).

b. Proiezione sull'utilizzo di biocarburanti. (Fonte: BP Energy Outlook, 2013). 
Il problema di analizzare la sufficienza delle risorse naturali disponibili a soddisfare una popolazione crescente è stato già affrontato in precedenza. Alla fine del XVIII secolo Malthus (1798) osservò che il tasso di crescita demografica era maggiore di quello della produzione alimentare, prevedendo quindi che, nel lungo periodo, l'umanità non avrebbe più avuto risorse sufficienti per sfamare se stessa. Nelle sue analisi Malthus non considerava il notevole aumento di capacità di accesso al cibo, oltre che di produzione dello stesso, che sarebbe stato prodotto dai progressi tecnologici. La storia moderna e contemporanea è stata infatti contraddistinta dall'avvento di almeno tre importanti "rivoluzioni" che hanno aumentato la produzione alimentare: (1) la rivoluzione industriale agli inizi del 1800, (2) la così detta "rivoluzione verde" negli anni Cinquanta, e (3) l'intensificazione del commercio mondiale. Negli ultimi due decenni il trade (commercio) di cibo, ha reso possibile la crescita demografica di nazioni non aventi le risorse naturali domestiche sufficienti a soddisfare l'incremento della domanda di cibo. Va sottolineato come il trade di cibo sia in realtà un importexport di acqua virtuale (Allan, 1998), di quell'acqua cioè che è stata necessaria per produrre tale cibo. Da ciò discende quindi come nazioni affette da scarsità idrica di tipo fisico abbiano potuto sostenere una crescita demografica senza incorrere in problemi si malnutrizione.

L'effetto del trade di acqua virtuale può essere spiegato tramite il bilancio idrico virtuale che per semplicità possiamo pensare alla scala nazionale. In tale analisi la disponibilità della risorsa idrica rinnovabile viene determinata dal clima, mentre la domanda idrica, che poi per noi è una domanda di cibo declinata in termini di acqua, viene definita dalla numerosità di popolazione e il tipo di alimentazione che la detta.

Se la domanda di cibo, e quindi di acqua, è maggiore della disponibilità dello stesso, noi ci troviamo in una situazione che è di stress idrico (cibo). A questo stress idrico è possibile rispondere in due modi. Se la nazione non è in grado di compensare tale deficit, non ha cioè la capacità economica per risolvere questo problema, il consumo (acqua, cibo) resta al di sotto di quella che è la domanda, lo stress permane potendosi trasformare in una situazione di malnutrizione. Qualora invece la situazione di stress viene superata tramite l'importazione, il consumo viene eguagliato alla domanda. Si innesca quindi un commercio di beni fra nazioni aventi risorse naturali per produrli e nazioni aventi risorse economiche per acquistarle. Al commercio di beni è quindi associato un commercio di risorse naturali, in modo particolare di acqua, la cosiddetta acqua virtuale. 
Grazie a tale commercio nazioni affette da scarsità idrica di tipo fisico (es. i paesi medio orientali) hanno potuto sostenere l'aumento di popolazione osservato negli ultimi decenni (Suweis et al., 2013). La Fig. 6a mostra la rete degli scambi commerciali espressa in termini di acqua virtuale e Fig. $6 b$ mostra il grande incremento di tale commercio osservato nell'ultimo decennio, quando, oltre ad avere un trade di acqua strettamente legato ai prodotti alimentari, si è avuto anche un trade di acqua virtuale legato a prodotti di energia, in particolare biocarburanti.
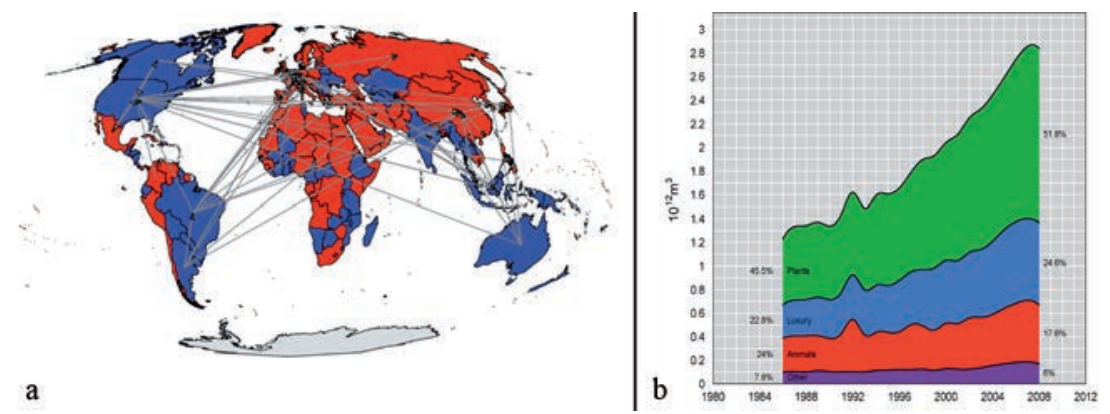

Fig. 6. a. Rete degli scambi commerciali. (Fonte: Carr et al., 2013).

b. Acqua virtuale associata agli scambi nel tempo. (Fonte: Carr et al., 2013).

Vi è un grande dibattito sulla possibilità di considerare il trade quale strategia a lungo termine per assicurare una sicurezza idrica e alimentare di una nazione.

Il problema principale nel considerare il trade quale strategia di lungo termine risiede nel considerare i beni soggetti a trade disconnessi dalle risorse naturali (risorse che non sono illimitate) necessarie alla loro produzione. Da ciò ne consegue che la crescita degli importatori è basata su di un import limitato solo dalla capacità economica, mentre quella degli esportatori è basata su tutte le risorse naturali disponibili (non depurate cioè delle risorse naturali necessarie a produrre i beni esportati). In caso di crescita sia degli importatori che degli esportatori, basata sulle medesime risorse naturali, si potrebbe avere una crisi in termini di risorse naturali creando una situazione di disequilibrio fra domanda e offerta di beni (e.g. prodotti agricoli). Tale disequilibrio, inoltre, potrebbe essere causato da altri fattori quali una situazione di shock alla produzione agricola (e.g. estremo idrologico quale siccità, alluvione etc.).

Quali strategie si stanno mettendo in atto per diminuire la dipendenza dal tade? 
La reazione alla crisi finanziaria e alimentare del 2008-2011 può darci qualche indicazione. Nel 2008 shock "climatici", nella fattispecie una siccità, hanno diminuito il raccolto in quelli che sono i "granai del mondo" (e.s. Ucraina) determinando una diminuzione di offerta di cereali sul mercato. Al contempo, le politiche energetiche in USA e UE, volte alla diminuzione degli effetti dei gas serra, hanno incrementato la domanda di prodotti agricoli da destinare alla produzione di energia (biocarburanti). Si è quindi osservato un grande disequilibrio fra un offerta di prodotti alimentari, diminuita rispetto agli anni immediatamente precedenti, e una domanda degli stessi, aumentata rispetto al recente passato. Ne è risultata una diminuzione delle esportazione da parte dei paesi colpiti dalla calamità naturale e un notevole incremento del prezzo del cibo accompagnato, a volte, da grossi disordini sociali.

In risposta governi e multinazionali hanno cominciato ad investire su grandi estensioni di terreni (LSLA) situati perlopiù in paesi in via di sviluppo in vista o di una produzione diretta dei beni attualmente importati o di una speculazione finanziaria. Tale fenomeno, qualora avvenga in violazione dei diritti umani, senza un processo decisionale trasparente e democratico, senza previo consenso dei preesistenti utilizzatori dei terreni, e senza considerazione degli impatti ambientali, viene chiamato in accezione negativa "land grabbing" (ILC, 2011). Dal 2008, quando è iniziato questo fenomeno, al 2016 sono stati acquisiti 45 milioni di ettari che equivalgono ad una superficie maggiore ( 1.5 volte) a quella della nostra penisola e più di cinque volte quella del Portogallo. Tali acquisizioni sono presenti in tutti i continenti eccetto l'Antartide e concentrate maggiormente in Africa ( 49\%) e in Asia \& Oceania (41\%) (Fig. 7).

A tali acquisizioni è associato un utilizzo totale di risorsa idrica suddivisa in verde, che indica l'acqua di precipitazione, e in blu, quella d'irrigazione, e in grigio, che è l'acqua che serve ad abbattere il carico inquinante prodotto dai fertilizzanti che vengono utilizzati in queste terre. La risorsa idrica dolce utilizzata per la coltivazione di queste terre è pari a a 487 milioni di metri cubi dove 380 milioni di metri cubi sono di acqua verde, 75 di acqua blu e 32 milioni di metri cubi di acqua grigia. Tale quantità equivale a quasi 10 volte il volume del lago di Garda (50 milioni di metri cubi). E' interessante notare che se mentre per ciò che attiene la terra il continente che mostra la maggiore estensione areale di terreno acquisito è l'Africa, per ciò che attiene la risorsa idrica esso è Asia\&Oceania, in dipendenza della esigenza 
idrica della tipologia di colture coltivate in tali continenti. I paesi ove avviene la maggiore acquisizione di risorsa idrica sono la Malesia, l'Indonesia, la Papua Nuova Guinea dove le acquisizioni di terreno sono principalmente rivolte alla coltivazione di palma da olio e canna da zucchero (colture molto utilizzate anche nel settore dei biocarburanti), mentre in Africa il Sudan e il sud Sudan. Alla scala globale la maggior quantità di acqua acquisita è di tipo verde benché il 15\% sia di irrigazione.

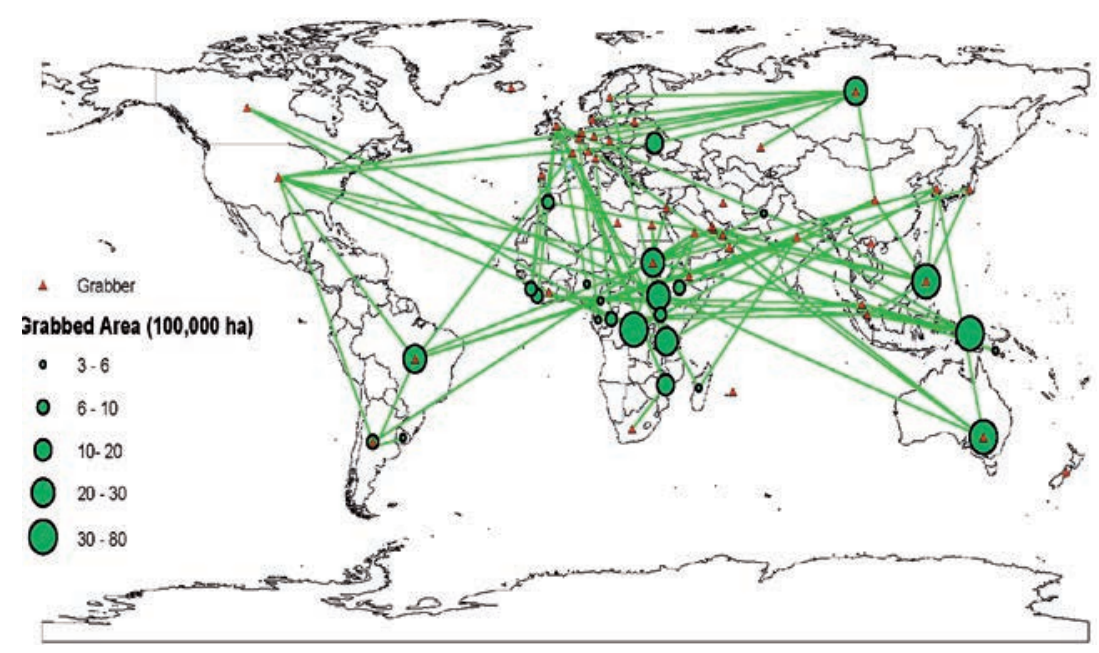

Fig. 7. Aree oggetto di acquisizione (cerchio verde) ed investitori (triangolo rosso). (Fonte: Rulli et al., 2013).

Tale acqua di irrigazione è dovuta quasi esclusivamente alle terre acquisite in Africa e rappresenta l'acqua di irrigazione necessaria ad una ottimale crescita delle colture oggetto di coltivazione. Va sottolineato come in molte zone dell'Africa non siano a tutt'oggi presenti le infrastrutture necessari per l'irrigazione di tali terre (Fig. 8).

L'analisi della produzione agricola in queste terre secondo le tecniche di produzione attualmente presenti ha evidenziato come, qualora coltivate, tali terre sarebbero in grado di nutrire 200 milioni persone fornendo ad esse un contenuto calorico giornaliero pari a $2400 \mathrm{kcal}$. Il miglioramento delle tecniche agricole, di gestione del suolo e dell'irrigazione consentirebbero un aumento della resa agricola fino alla chiusura del cosiddetto gap colturale. In tal caso le persone nutribili sareb- 
bero 300 milioni che equivalgono a più di un terzo delle persone attualmente affette da malnutrizione (Fig. 9).
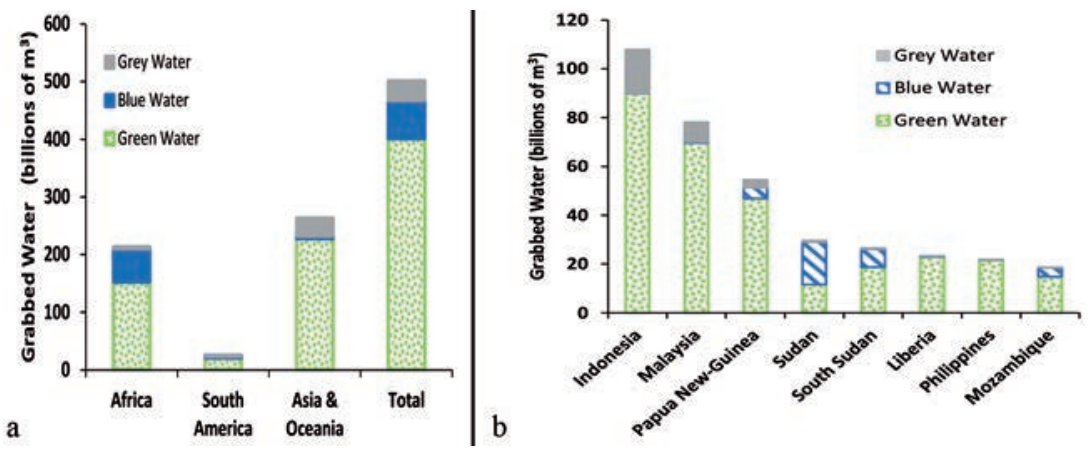

Fig. 8. Risorsa idrica associata alla coltivazione delle terre oggetto di investimento alla scala di continente (a) e riportata per i 10 paesi maggiormente coinvolti nel processo di investimento. (Fonte: Rulli e D'Odorico, 2013).

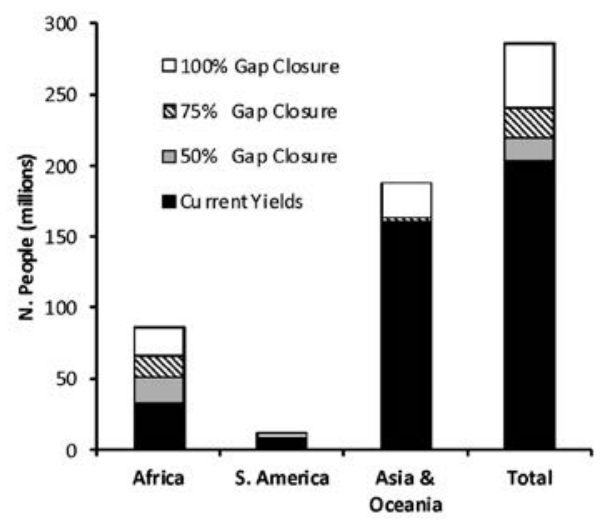

Fig. 9. Persone nutribili con il cibo prodotto nelle terre oggetto di investimento straniero per diversi livelli di miglioramento della resa agricola.

(Fonte: Rulli e D'Odorico, 2013).

Va sottolineato come attualmente la produzione delle LSLA venga destinata o al paese investitore o al mercato internazionale, inaccessibile quindi alle popolazioni autoctone ed esacerbando, di conseguenza, le condizioni di insicurezza alimentare. Da quanto sopra riportato si evince come la corsa alla terra alla quale stiamo assistendo negli ultimi anni da parte di governi, società, fondi di investimento sia asso- 
ciata ad una corsa per l'appropriazione anche di altre risorse ambientali, in primis l'acqua, risorsa basilare per la produzione agricola e quindi per la produzione di cibo e anche di energia. L'acquisizione di terre non è solo una corsa per la terra, ma anche una corsa per le risorse di acqua dolce disponibili in essa.

Le grandi acquisizioni di terra, qualora fossero gestite secondo un criterio win-win per tutti gli attori coinvolti, potrebbero invece rappresentare un'opportunità per aumentare la produzione agricola in vista dell'imminente crescente bisogno di essa. Terre attualmente poco produttive a causa della mancanza di tecnologie e conoscenza di appropriate tecniche di gestione del suolo e dell'acqua potrebbero beneficiare di un apporto tecnologico e conoscitivo da parte degli investitori.

\section{BIBLIOGRAFIA}

Allan, J.A., Virtual water: A strategic resource global solutions to regional deficits, Ground Water, 36(4), 545-546, doi:10.1111/j.1745-6584.1998.tb0282, 1998.

Antonelli, M., G Siciliano, ME Turvani, MC Rulli (2015). Global investments in agricultural land and the role of the EU: Drivers, scope and potential impacts. LAND USE POLICY, vol. 47, p. 97-111, ISSN: 0264-8377, doi: 10.1016/j.landusepol.2015.04.007

Alexandratos, N. and J. Bruinsma. (2012). World agriculture towards 2030/2050: the 2012 revision. ESA WORKING PAPER No. 12-03. Rome, FAO.

Boserup E., Population and Technological Change, (Univ. of Chicago Press, Chicago), 1981.

Brown ME, et al. Country and regional staple food price indices for improved identification of food insecurity. Glob Environ Change 22(3):784-794, 2012.

Carr JA, D'Odorico P, Laio F, Ridolfi L (2013). Recent History and Geography of Virtual Water Trade. PLoS ONE8(2): e55825. https://doi.org/10.1371/journal. pone. 0055825

Chiarelli D.D., Davis K.F., Rulli M.C., D'Odorico P. (2016). Climate change and largescale land acquisitions in Africa: Quantifying the future impact on acquired water resources ADVANCES IN WATER RESOURCES, 94, 231-237 10.1016/j.advwatres.2016.05.016

Cohen, J.E., How Many People Can the Earth Support? Norton, New York, 1995.

Davis, K.F., P. D'Odorico, M. Rulli (2014). Land grabbing: a preliminary quantification of economic impacts on rural livelihoods. POPULATION AND ENVIRONMENT, vol. 36, p. 180-192.

Davis, K.F., P. D'Odorico, M.C. Rulli (2014). Moderating diets to feed the future. EARTH'S FUTURE, vol. 2, p. 559-565, ISSN: 2328-4277, doi: 10.1002/ 2014EF000254 
Davis, K.F., M.C. Rulli, and P. D'Odorico (2015). The global land rush and climate change, EARTH'S FUTURE, 3, 298-311, doi:10.1002/2014EF000281.

Davis K.F., Yu K., Rulli M.C., Pichdara L., D’Odorico P. (2015). Accelerated deforestation driven by large-scale land acquisitions in Cambodia. NATURE GEOSCIENCE, vol. 8, p. 772-775, ISSN: 1752-0894, doi: 10.1038/ngeo2540

Davis K.F., Rulli M.C., Garrassino F., Chiarelli D., Seveso A., D’Odorico P. (2017) Water limits to closing yield gaps. ADVANCES IN WATER RESOURCES 99, 67-75. http://dx.doi.org/10.1016/j.advwatres.2016.11.015

Davis K.F., Seveso A., Rulli M.C., D’Odorico P. (2017). Water Savings of Crop Redistribution in the United States. WATER 2017, 9(2), 83; doi:10.3390/ w9020083.

Davis K.F., Rulli M.C., Seveso A., D’Odorico P. (2017). Increased food production and reduced water use through optimized crop distribution. NATURE GEOSCIENCE, doi:10.1038/s41561-017-0004-5.

Dell'Angelo J., D'Odorico P., Rulli M.C., Marchand P. (2016). The Tragedy of the Grabbed Commons: Coercion and Dispossession in the Global Land Rush. WORLD DEVELOPMENT, vol.92, pp1-12, http://dx.doi.org/10.1016/j. worlddev.2016.11.005.

D'Odorico, P., M.C. Rulli (2014). The land and its people. NATURE GEOSCIENCE, vol. 7, p. 324-325.

D’Odorico, P., Laio, F. \& Ridolfi, L. (2010). Does globalization of water reduce societal resilience to drought? GEOPHYS. RES. LETT. 37, L13403.

D'Odorico, P., Rulli, M.C. (2013). The fourth food revolution. NATURE GEOSCIENCE, vol. 6, p. 417-418, ISSN: 1752-0894, doi: 10.1038/ngeo1842

D’Odorico P., Rulli, M.C., Dell'Angelo J., Davis K.F., (2017). New frontiers of land and water commodification: socioenvironmental controversies of large-scale land acquisitions, LAND DEGRAD. DEVELOP. DOI: 10.1002/ldr.2750.

Fader M., Rulli, M.C. et al. (2016). Past and present biophysical redundancy of countries as a buffer to changes in food supply ENVIRONMENTAL RESEARCH LETTERS 11 (5), 055008 doi: 10.1088/1748-9326/11/5/055008.

Falkenmark, M. J., J. Rockström, and H. Savenjie (2004). Balancing Water for Humans and Nature, EARTHSCAN, LONDON.

Foley JA, et al., Solutions for a cultivated planet. NATURE 478:337-342, 2011.

Food and Agriculture Organization of the United Nations (FAO), World Agriculture: Towards 2015/2030. A FAO Perspective. FOOD AND AGRICULTURE ORGANIZATION OF THE UNITED NATIONS/EARTHSCAN, ROME, Italy/USA, 2013.

Godfray, H.C.G. Food for Thought, Proc. Natnl Acad. Sci, USA, PNAS, doi: 10.1073/pnas.1118568109, 2011.

Hoekstra A.Y., M.M. Mekonnen (2011). The water footprint of humanity PROCEEDINGS OF THE NATIONAL ACADEMY OF SCIENCES OF THE UNITED STATES OF AMERICA, 109 (2012), pp. 3232-3237.

International Energy Agency (IEA). (2013). Toward a sustainable energy future. http://www.iea.org/ textbase/nppdf/free/2013/future2013.pdf 
Liu JG, Yang H, Savenije HH. China's move to higher-meat diet hits water security. Nature 454(7203): 397, 2008.

Malthus T. An Essay on the Principle of Population (J. Johnson, London), 1798.

Marchand P., Rulli M.C. et al. (2016). Reserves and trade jointly determine exposure to food supply shocks, ENVIRONMENTAL RESEARCH LETTERS, 11 (9), 095009. doi:10.1088/1748-9326/11/9/095009.

Rockstrom, J., M. Falkenmark, M. Lannerstad, and L. Karlberg, The planetary water drama: Dual task of feeding humanity and curbing climate change, Geophys. Res. Lett., doi:10.1029/2012GL051688, 2012.

Rulli M.C., Bellomi D., Cazzoli A., De Carolis G., D’Odorico P. (2016). The waterland-food nexus of first-generation biofuels. SCIENTIFIC REPORTS, 6, Art. num. 22521, doi:10.1038/srep22521.

Rulli, M.C., P. D'Odorico (2014). Food appropriation through large scale land acquisitions. ENVIRONMENTAL RESEARCH LETTERS, vol. 9, p. 1-8, ISSN: 1748 9326, doi: 10.1088/1748-9326/9/6/064030.

Rulli M.C., Saviori A., D'Odorico P. (2013). Global land and water grabbing. PROCEEDINGS OF THE NATIONAL ACADEMY OF SCIENCES OF THE UNITED STATES OF AMERICA, vol. 110, p. 892-897, doi: 10.1073/ pnas. 1213163110.

Rulli M.C., P. D'Odorico (2013). The water footprint of land grabbing. GEOPHYSICAL RESEARCH LETTERS, vol. 40, p. 1-6, ISSN: 0094-8276, doi: 10.1002/ 2013 GL058281.

Suweis, S., A. Rinaldo, A. Maritan, and P. D'Odorico. Water-controlled wealth of nations, Proc. Natnl Acad. Sci, USA, PNAS, doi: 10.1073/pnas.1222452110, 2013.

United Nations-Department of economic and social affairs-Population division, World population prospects: the 2012 revision. http://esa.un.org/wpp/ 